\title{
Impactos da polifarmácia na saúde bucal de idosos: um protocolo de revisão de
}

\section{escopo}

\author{
Impacts of polypharmacy on the oral health of the elderly: a scoping review protocoltherapy \\ Impactos de la polifarmacia en la salud bucal de los ancianos: un protocolo de revisión del alcance
}

Recebido: 27/10/2021 | Revisado: 06/11/2021 | Aceito: 10/11/2021 | Publicado: 15/11/2021

Gabrieli Duarte Farias

ORCID: https://orcid.org/0000-0001-6904-2856 Universidade Federal da Paraíba, Brasil

E-mail: gabrieli.duarte@academico.ufpb.br

Adriça Gondim De Lima

ORCID: https://orcid.org/0000-0003-1607-2351 Universidade Federal da Paraíba, Brasil E-mail: adricagondim@ hotmail.com

Rodrigo Carneiro Meira Lima

ORCID: https://orcid.org/0000-0001-7018-6179 Universidade Federal da Paraíba, Brasil E-mail: rodrigo.cml2@hotmail.com

Wandeberg Patrick Morais da Silva

ORCID: https://orcid.org/0000-0003-2505-2985 Universidade Federal da Paraíba, Brasil E-mail: freirewandeberg@gmail.com

Eduarda Gomes Onofre de Araújo ORCID: https://orcid.org/0000-0001-7107-6107 Universidade Federal da Paraíba, Brasil

E-mail: eduarda.onofre@academico.ufpb.br Júlio César Guimarães Freire

ORCID: https://orcid.org/0000-0002-4287-3278 Universidade Federal da Paraíba, Brasil

E-mail: julio.guimaraes@academico.ufpb.br

Rilary Rodrigues Feitosa ORCID: https://orcid.org/0000-0003-4970-3498 Universidade Federal da Paraíba, Brasil

E-mail: rilary.feitosa@academico.ufpb.br

Carmem Silvia Laureano Dalle Piagge ORCID: https://orcid.org/0000-0001-7999-2943 Universidade Federal da Paraíba, Brasil

E-mail: carmem.piagge@ academico.ufpb.br

Cláudia Batista Mélo

ORCID: https://orcid.org/0000-0001-5300-3510 Universidade Federal da Paraíba, Brasil

E-mail: claudia.melo@academico.ufpb.br

\section{Resumo}

A polifarmárcia é o termo aplicado para referir-se ao uso de múltiplas combinações medicamentosas e, atualmente, tem recebido muita atenção devido aos inúmeros impactos à saúde e à frequência com a qual é utilizada. Dessa forma, a polifarmácia é um problema crítico, passível de atingir qualquer pessoa, sendo mais danosa para os idosos devido aos potenciais riscos de eventos adversos e colaterais, que podem influenciar na saúde sistêmica e bucal. O objetivo desse protocolo é apresentar etapas metodológicas para realização de uma revisão de escopo, a fim de explorar os impactos da polifarmácia na saúde bucal de idosos, mapeando e sumarizando as evidências quanto às manifestações e quadros patológicos mais recorrentes devido à condição estudada, além de verificar como os estudos vêm sendo conduzidos e identificar lacunas na literatura. Esse protocolo foi desenvolvido de acordo com as recomendações do Manual do Instituto Joanna Briggs e registrado na Open Science Framework. Serão utilizadas as bases de dados PubMed via MEDLINE, Science Direct (Elsevier), Web of Science, Scopus, LILACS e Google Scholar para busca dos estudos. Os dados serão extraídos e apresentados na forma de quadros e/ou imagens e de forma descritiva, conforme indicado no checklist proposto pelo PRISMA-ScR. Espera-se que esse protocolo possibilite a realização de uma revisão de escopo, com metodologia consistente e, a partir dela, a obtenção de um maior conhecimento acerca dos impactos da polifarmácia na saúde bucal de idosos, ampliando a visão que se tem sobre o tema.

Palavras-chave: Polifarmácia; Manifestações bucais; Saúde bucal. 


\begin{abstract}
Polypharmacy is the term used to refer to the use of multiple drug combinations and has received much attention due to its numerous health impacts and the frequency with which it is used. Thus, polypharmacy is a critical problem that can affect anyone, being more harmful to the elderly due to the potential risks of adverse and collateral events, which can influence oral and systemic health. The objective of this protocol is to present methodological steps for conducting a scoping review in order to explore the impacts of polypharmacy on the oral health of the elderly, mapping and summarizing the evidence on the most recurrent manifestations and pathological conditions due to the condition studied, in addition to verifying how studies have been conducted and identifying gaps in the literature. This protocol was developed according to the recommendations of the Joanna Briggs Institute Manual and registered in the Open Science Framework. PubMed via MEDLINE, Science Direct (Elsevier), Web of Science, Scopus, LILACS, and Google Scholar will be used to search the studies. The data will be extracted and presented in the form of tables and/or images and in descriptive form, as indicated in the checklist proposed by PRISMA-ScR. It is expected that this protocol will enable the realization of a scoping review, with consistent methodology and, from it, the attainment of greater knowledge about the impacts of polypharmacy on the oral health of the elderly, broadening the vision that we have on the subject.
\end{abstract}

Keywords: Polypharmacy; Oral manifestations; Oral health.

\title{
Resumen
}

La polifarmacia es el término que se aplica para referirse al uso de múltiples combinaciones de medicamentos y, en la actualidad, ha recibido mucha atención debido a los numerosos impactos en la salud y a la frecuencia con la que se utiliza. Así, la polifarmacia es un problema crítico, que puede afectar a cualquier persona, siendo más perjudicial para los ancianos debido a los riesgos potenciales de eventos adversos y colaterales, que pueden influir en la salud sistémica y bucodental. El objetivo de este protocolo es presentar los pasos metodológicos para llevar a cabo una revisión de alcance con el fin de explorar los impactos de la polifarmacia en la salud bucodental de los ancianos, mapeando y resumiendo la evidencia sobre las manifestaciones más recurrentes y las condiciones patológicas debidas a la condición estudiada, además de verificar cómo se han realizado los estudios e identificar las lagunas en la literatura. Este protocolo fue desarrollado según las recomendaciones del Manual del Instituto Joanna Briggs y registrado en el Open Science Framework. Para extraer los estudios se utilizarán las bases de datos PubMed vía MEDLINE, Science Direct (Elsevier), Web of Science, Scopus, LILACS y Google Scholar. Los datos se extraerán y se presentarán en forma de gráficos y/o imágenes y de forma descriptiva, como se indica en la lista de comprobación propuesta por PRISMA-ScR. Se espera que este protocolo permita la realización de una revisión de alcance, con una metodología consistente y, a partir de ella, la consecución de un mayor conocimiento sobre los impactos de la polifarmacia en la salud bucodental de las personas mayores, ampliando la visión que se tiene sobre el tema.

Palabras clave: Polifarmacia; Manifestaciones orales; Salud oral.

\section{Introdução}

Em menos de dois séculos, a humanidade aumentou expressivamente a sua expectativa de vida que, consequentemente, causou um impacto significativo no contingente de idosos (Carmona \& Simões, 2020). O envelhecimento populacional está relacionado com a redução dos números de natalidade, da fecundidade e da mortalidade infantil (Costa, Oliveira, \& Novaes, 2017). Os avanços médico-tecnológicos para tratar e prevenir doenças, assim como as melhorias das condições de saneamento básico foram cruciais para desencadear o aumento da expectativa de vida (Pereira, et al., 2017; Souza, et. al., 2018). Em geral, essas mudanças culminaram em uma população que vive mais anos, sendo esse processo gradativo conhecido como "transição epidemiológica", o qual gera a necessidade de estudos focados na qualidade de vida dos idosos (Borges, et al., 2017).

De acordo com a Organização Mundial da Saúde (OMS), são consideradas idosas, as pessoas com idade acima de 60 anos e residentes em países em desenvolvimento e, acima de 65 anos, quando vivem em países desenvolvidos (World Heatlh Organization, 2005). Em 2019, a Organização das Nações Unidas (ONU) lançou o relatório World Population Prospects 2019, onde é possível observar que o número de brasileiros idosos de 60 anos ou mais em 1950 era de 2,6 milhões, crescendo para 29,9 milhões em 2020 e estima-se que alcançará 72,4 milhões em 2100 (United Nations, 2019).

Com a mudança do perfil da população, surge a necessidade de garantir uma assistência adequada a esses idosos, sobretudo, no que concerne ao principal fator da longevidade que é a saúde da população geriátrica (Freitas, et.al., 2019; Paiva, 
Hillesheim, \& Haas, 2019). No Brasil, a saúde do idoso foi estabelecida como uma das seis prioridades pactuadas pelas três esferas do governo, através do Pacto pela Saúde, no qual destaca-se o papel vital da saúde bucal do idoso (Brasil, 2006).

Com o envelhecimento, algumas doenças crônicas, geralmente associadas à idade, como dislipidemia, cardiopatia, diabetes, depressão e doenças infecciosas começam a surgir, causando a necessidade da utilização de múltiplos medicamentos, a qual se dá o nome de polifarmácia (Rodrigues \& Oliveira, 2016; Carvalho, et al., 2020). Essa condição pode ser compreendida pelo uso de 5 ou mais tipos de medicamentos de forma simultânea e que acontece, tipicamente, com indivíduos com mais de 60 anos (Gomes, 2017). Em um estudo realizado por norte-americanos, concluiu-se que mais de 40\% dos idosos com 65 anos ou mais usam 5 ou mais medicamentos e 12\% usam 10 ou mais medicamentos diferentes (Gurwitz, et al., 2003). Contudo, a grandeza e delimitação das consequências da polifarmácia entre os idosos ainda é pouco conhecida na maioria dos países (Rodrigues \& Oliveira, 2016).

Apesar da polifarmácia ser um objeto de estudo com relevância crescente na academia, ainda não existe um consenso na literatura que trate categoricamente a sua definição (Da Gama, 2019). Através da ótica quantitativa, grande parte dos artigos encontrados define a polimedicação como a prescrição de cinco ou mais classes de fármacos por doente. Também se encontra o conceito de polifarmácia excessiva ou hiperpolifarmácia, que é a condição de medicação com número de fármacos superior a dez (Almeida, et al., 2017). No tocante à concepção qualitativa, tem-se a prescrição apropriada ou inapropriada. A última diz respeito à medicação desnecessária, ao uso de medicação que seria igual para a mesma doença, a medicação com efeitos adversos entre si e todos e quaisquer tipos de prescrições incorretas (Alves \& Ceballos, 2018).

A respeito dos efeitos da polifarmácia, comprova-se que tal prática aumenta a administração inadequada de drogas e, algumas vezes, leva ao consumo excessivo de medicamentos, favorecendo às ocorrências de erro de medicação, interações medicamentosas e reações adversas, resultando em má qualidade de vida (Ramos, et al., 2016; Carneiro, et al., 2018). Além desses efeitos prejudiciais à saúde, na maioria das vezes, a polifarmácia também pode afetar, de forma direta, as condições financeiras do idoso ou, em alguns casos, de seus familiares (Lopes, 2000).

Nesse contexto, o objetivo desse trabalho é apresentar um protocolo de revisão de escopo que visa explorar sistematicamente a literatura quanto aos impactos da polifarmácia na saúde bucal de idosos, mapeando e sumarizando as evidências quanto às manifestações e quadros patológicos mais recorrentes devido à condição estudada. Além disso, propõe verificar como os estudos vêm sendo conduzidos e identificar lacunas na literatura.

\subsection{Pergunta de Pesquisa}

Para formulação da pergunta de pesquisa, utilizou-se o mnemônico PCC (Paciente, Conceito e Contexto) para estudos qualitativos, baseado no Manual do Joanna Briggs Institute (JBI) (Peters, et al., 2020a). Desse modo, foram definidos como determinantes de interesse do estudo: População (P): idosos; Conceito (C): manifestações bucais e Contexto (C): uso de polifarmácia. A partir de tais determinantes, elaborou-se a seguinte questão norteadora da pesquisa: "Quais são os impactos da polifarmácia na saúde bucal de idosos?”. No Quadro 1, observa-se a estratégia PCC que será utilizada na revisão de escopo. 
Quadro 1. Estratégia PCC.

\begin{tabular}{|c|c|c|c|}
\hline \multirow{2}{*}{ Problema } & \multicolumn{3}{|c|}{ Quais são os impactos da polifarmácia na saúde bucal de idosos? } \\
\hline & População & Conceito & Contexto \\
\hline Extração & idosos & manifestações bucais & uso de polifarmácia \\
\hline Conversão & aged & oral manifestations & polypharmacy \\
\hline Combinação & $\begin{array}{l}\text { aged; elderly; elderlies; } \\
\text { health of the elderly }\end{array}$ & $\begin{array}{l}\text { oral manifestations; oral } \\
\text { manifestation; oral } \\
\text { health; pathology, oral }\end{array}$ & $\begin{array}{l}\text { polypharmacy; } \\
\text { polymedication }\end{array}$ \\
\hline Construção & $\begin{array}{l}\text { "aged" OR "elderly" OR } \\
\text { "elderlies" OR "health of } \\
\text { the elderly" }\end{array}$ & $\begin{array}{l}\text { "oral manifestations" OR } \\
\text { "oral manifestation" OR } \\
\text { "oral health" OR } \\
\text { "pathology, oral" }\end{array}$ & $\begin{array}{l}\text { "polypharmacy" OR } \\
\text { "polymedication" }\end{array}$ \\
\hline Uso da Estratégia & \multicolumn{3}{|c|}{$\begin{array}{l}\text { ("aged" OR "elderly" OR "elderlies" OR "health of the elderly") AND ("oral } \\
\text { manifestations" OR "oral manifestation" OR "oral health" OR "pathology, } \\
\text { oral") AND ("polypharmacy" OR "polymedication") }\end{array}$} \\
\hline
\end{tabular}

Fonte: Autores (2021).

\subsection{Critérios de Inclusão}

\subsubsection{População}

Serão incluídos estudos realizados com pacientes idosos (65 anos ou mais) que fazem uso de polifarmárcia, sem qualquer distinção de sexo ou etnia.

\subsubsection{Conceito}

O conceito de interesse proposto para a revisão de escopo é a busca de evidência científica sobre as manifestações orais em idosos que fazem uso de polifarmácia. Desse modo, serão consideradas as pesquisas que analisaram os efeitos à saúde bucal relacionados ao uso de vários medicamentos administrados de forma simultânea, independentemente do modo de apresentação e da posologia adotada.

\subsubsection{Contexto}

O contexto a ser considerado na revisão de escopo será aberto, pois serão incluídas fontes de evidência independente da configuração contextual dos participantes.

\subsubsection{Tipos de fontes de evidência}

O método de revisão de escopo proposto permitirá a consulta de várias fontes científicas, portanto, serão considerados estudos observacionais analíticos, incluindo ensaios clínicos, estudos de coorte, transversais, caso-controle e observacionais. Além disso, serão incluídas fontes adicionais provenientes da literatura cinzenta.

\section{Metodologia}

Trata-se de um protocolo para condução de um estudo do tipo revisão de escopo. No processo de elaboração, utilizaram-se as recomendações de estruturação do Manual do Instituto Joanna Briggs - JBI Manual for Evidence Synthesis (Peters, et al., 2020b). Para garantir a visibilidade e a transparência do processo de revisão, o protocolo foi reg istrado na Open 
Science Framework (https://osf.io/) com DOI: https://doi.org/10.17605/OSF.IO/FU5WC.

\subsection{Estratégia de busca}

Com o intuito de obter uma visão geral do estado atual do conhecimento, foi elaborada uma estratégia de busca, seguindo três passos, conforme orientações do Manual para revisões de escopo do JBI. Inicialmente, será realizada uma busca genérica nas bases PubMed e Web of Science por meio da testagem de termos MeSH e termos índices, na qual serão analisados os títulos e resumos dos artigos recuperados com o intuito de identificar termos que possam ser adicionados à estratégia de busca. Em seguida, a estratégia de busca será adaptada às demais bases de dados, de acordo com as particularidades de cada uma. No terceiro e último passo, será realizada uma busca manual na lista de referências dos artigos incluídos com a finalidade de identificar algum estudo de interesse.

\subsection{Fontes de informações}

A identificação dos estudos será realizada através das seguintes bases de dados: PubMed via MEDLINE, Science Direct (Elsevier), Web of Science, Scopus, LILACS (Literatura Latino Americana e do Caribe em Ciências da Saúde), bem como na literatura cinzenta por meio do Google Scholar.

\subsection{Seleção dos estudos}

Os estudos obtidos como resultado da busca nas bases de dados supracitadas serão exportados para o gerenciador de referências EndNote (Clarivate Analytics, USA), para remoção automática dos estudos duplicados e, em seguida, serão exportados para o Software Rayyan (Qatar Computing Research Institute), onde serão desenvolvidas as etapas de exclusão dos estudos, primeiro por leitura do título e resumo, depois por leitura na íntegra.

As etapas serão realizadas por dois revisores de forma independente e qualquer divergência quanto à exclusão dos artigos, será consultado um terceiro revisor. O detalhamento de todo processo será exibido na versão final da revisão de escopo, através de um fluxograma adaptado do Checklist PRISMA-ScR (Preferred Reporting Items for Systematic Reviews and Meta-Analyses extension for Scoping Reviews) (Tricco et al., 2018) (Figura 1). 
Figura 1. Fluxograma das etapas de busca e seleção dos estudos incluídos na revisão de escopo.

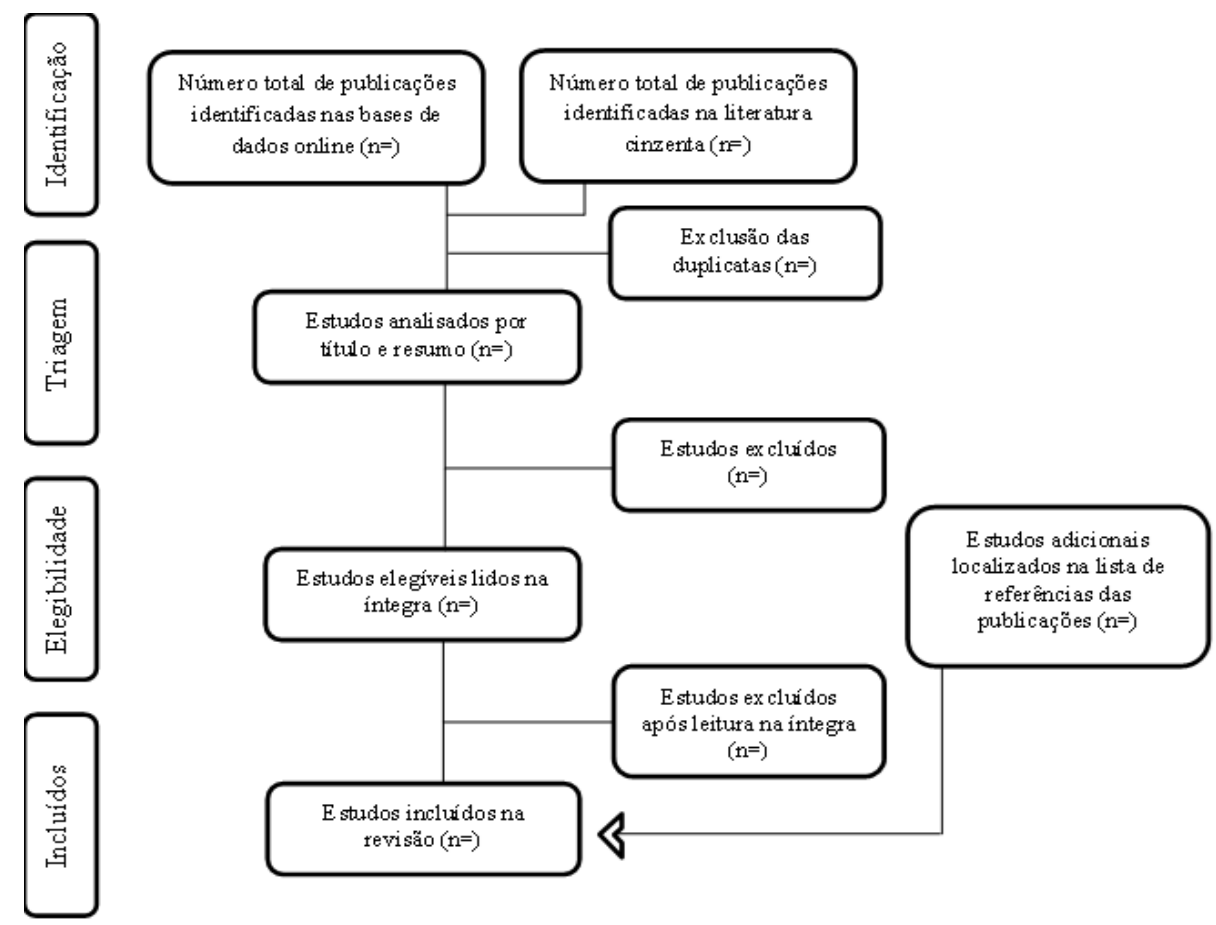

Fonte: Adaptado do Preferred Reporting Items for Systematic Reviews and Meta-Analyses extension for Scoping Reviews (PRISMA-ScR) Moher et al., (2015).

\subsection{Extração de Dados}

A etapa de extração dos dados também será realizada por dois revisores de maneira independente, previamente orientados e treinados, a fim de organizar, analisar e interpretar as informações disponíveis nos estudos selecionados de acordo com o objetivo. Para esse procedimento, será estruturado pelos próprios autores um formulário de extração de dados, contendo: autores, ano de publicação, país, delineamento do estudo, objetivo(s), amostra e as principais manifestações orais em idosos com polifarmácia.

Um teste piloto, utilizando cinco artigos, será conduzido com o objetivo de verificar a concordância entre os revisores no preenchimento do formulário. Se necessário, ajustes poderão ser realizados no formulário para garantir a melhor obtenção dos dados.

\subsection{Apresentação dos dados}

Os dados obtidos serão sintetizados e exibidos através de tabelas e/ou quadros, aliados a descrição narrativa, objetivando facilitar a compreensão do leitor quanto ao tópico de pesquisa.

\section{Considerações Finais}

A criação do referido protocolo possibilitará a realização de uma revisão de escopo, metodologicamente embasada nos princípios estabelecidos pelo manual do Instituto Joanna Briggs e, a partir dela, a obtenção de um maior conhecimento acerca dos impactos da polifarmácia na saúde bucal de idosos.

Para trabalhos futuros, sugere-se a elaboração de outros protocolos de revisão, com métodos objetivos e bem definidos, que possibilitem uma visão ampla sobre a temática das manifestações de lesões orais decorrentes do uso de polifarmácia em idosos. 


\section{Referências}

Almeida, N. A. D., Reiners, A. A. O., Azevedo, R. C. D. S., Silva, A. M. C. D., Cardoso, J. D. C., \& Souza, L. C. D. (2017). Prevalência e fatores associados à polifarmácia entre os idosos residentes na comunidade. Revista Brasileira de Geriatria e Gerontologia , 20, $138-148$.

Alves, N. M. C., \& de Ceballos, A. G. D. C. (2018). Polifarmácia em idosos do programa universidade aberta à terceira idade. Journal of Health \& Biological Sciences, 6(4), 412-418.

Borges, E., et al. O envelhecimento populacional: um fenômeno mundial. O envelhecimento populacional um fenômeno, p. 17, 2017.

Brasil. (2006). Portaria No 399. Divulga o Pacto pela Saúde. Consolidação do SUS e aprovação as Diretrizes Operacionais do Referido Pacto. Diário Oficial da União. https://bvsms.saude.gov.br/bvs/saudelegis/gm/2006/prt0399_22_02_2006.html

Carmona, M. J. C., \& Simões, C. M. (2020). Paciente 4.0-O desafio do cuidado ao paciente idoso. Revista Brasileira de Anestesiologia. 70(1): 1-2.

Carneiro, J. A., Ramos, G. C. F., Barbosa, A. T. F., Medeiros, S. M., de Almeida Lima, C., da Costa, F. M., \& Caldeira, A. P. (2018). Prevalência e fatores associados à polifarmácia em idosos comunitários: estudo epidemiológico de base populacional. Medicina (Ribeirão Preto), 51 (4), $254-264$.

Carvalho, G. A. O., Carvalho, N. S., Sousa, G. P., Lima, D. E. O., Matos, A. F. B., Silva, F. A. D. J. C., \& Sousa Bezerra, W. B. (2020). Manifestações bucais advindas da polifarmácia em idosos de um abrigo público de Teresina-Piauí. Research, Society and Development, 9(7), e08973522-e08973522.

Costa, G. M., Oliveira, M. L. C., \& Novaes, M. R. C. G. (2017). Fatores associados à polifarmacoterapia entre idosos assistidos pela estratégia saúde da família. Revista Brasileira de Geriatria e Gerontologia, 20(4), 528-537.

da Gama, M. F. R. O. (2019). Polifarmácia no idoso-Consequências, desafios e estratégias de abordagem.

Freitas, D. E. D., Martins, B. B., Paulino, J. K. B., Veríssimo, D. E. D. F., \& Belém, L. D. F. (2019). Polimedicação de idosos na universidade aberta à maturidade. Revista de Divulgação Científica Sena Aires, 8(3), 316-321.

Gomes, H. O., \& Caldas, C. P. (2016). Uso inapropriado de medicamentos pelo idoso: polifarmácia e seus efeitos. Revista Hospital Universitário Pedro Ernesto. 7(1): 1-12.

Gurwitz, J. H., Field, T. S., Harrold, L. R., Rothschild, J., Debellis, K., Seger, A. C., \& Bates, D. W. (2003). Incidência e evitabilidade de eventos adversos a medicamentos entre idosos em ambiente ambulatorial. Jama, 289 (9), 1107-1116.

Lopes, A. (2000). A Sociedade Brasileira de Geriatria e Gerontologia e os desafios da Gerontologia no Brasil.

Moher, D., Shamseer, L., Clarke, M., Ghersi, D., Liberati, A., Petticrew, M., \& Stewart, L. A. (2015). Preferred reporting items for systematic review and meta-analysis protocols (PRISMA-P) 2015 statement. Systematic reviews, 4(1), 1-9.

Paiva, K. M. D., Hillesheim, D., \& Haas, P. (2019). Atenção ao idoso: percepções e práticas dos Agentes Comunitários de Saúde em uma capital do sul do Brasil. In CoDAS (Vol. 31). Sociedade Brasileira de Fonoaudiologia.

Pereira, K. G., Peres, M. A., Iop, D., Boing, A. C., Boing, A. F., Aziz, M., \& d’Orsi, E. (2017). Polifarmácia em idosos: um estudo de base populacional. Revista Brasileira de Epidemiologia, 20, 335-344.

Peters, M. D. J., Godfrey, C., McInerney, P., Munn, Z., Tricco, A. C., \& Khalil, H. Chapter 11: Scoping Reviews (2020a). In: Aromataris E, Munn Z (Editors). JBI Manual for Evidence Synthesis, JBI, 2020. https://synthesismanual.jbi.global.

Peters, M., Marnie, C., Tricco, A. C., Pollock, D., Munn, Z., Alexander, L., McInerney, P., Godfrey, C. M., \& Khalil, H. (2020b). Updated methodological guidance for the conduct of scoping reviews. JBI evidence synthesis, 18(10), 2119-2126.

Ramos, L. R., Tavares, N. U. L., Bertoldi, A. D., Farias, M. R., Oliveira, M. A., Luiza, V. L., \& Mengue, S. S. (2016). Polifarmácia e polimorbidade em idosos no Brasil: um desafio em saúde pública. Revista de Saúde Pública, 50(2): 1-13.

Rodrigues, M. C. S., \& Oliveira, C. D. (2016). Interações medicamentosas e reações adversas a medicamentos em polifarmácia em idosos: uma revisão integrativa. Revista Latino-Americana de Enfermagem, 24, 1-17.

Souza, D. M., Souza, L. B., Lana, G. G., Souza, S. M., Aguilar, N. C., \& Silva, D. R. (2018). Uso inapropriado de medicamentos pelo idoso: polifarmácia e seus efeitos. Pensar Acadêmico, 16(2), 166-178.

Tricco, A. C., Lillie, E., Zarin, W., O'Brien, K. K., Colquhoun, H., Levac, D., Moher, D., Peters, M. D., Horsley, T., Weeks, L., \& Hempel, S., (2018). PRISMA extension for scoping reviews (PRISMA-ScR): checklist and explanation. Annals of internal medicine, 169(7), 467-473.

United Nations (2019). Department of Economic and Social Affairs. World Population Prospects. https://population.un.org/wpp/Download/Standard/Population/

World Health Organization. (2005). Envelhecimento ativo: uma política de saúde. 